\title{
In-vivo Experimental and Numerical Studies of Cardiac Flow in Right Atrium
}

The experimental and computational modes of determining cardiac flow are presented. In a computational fluid dynamics study, we attempt to simulate the physiological environment and flow conditions accurately in the right atrium. During the initial stage, we performed magnetic resonance imaging at multiple slices for chamber reconstruction and mesh refinements. A transient-state numerical simulation based on physiological data is developed. For our experimental approach, phase contrast magnetic resonance imaging of a selected slice of the heart is used to measure velocity of blood within the heart chamber in-vivo. This imaging technique can provide velocity maps to compare with the simulated flow. The velocity maps by the two methods are post-processed to generate vorticity maps for evaluation. Both experimental and computational results present the flow pattern of right atrial vortices with good agreement.

Keywords: Computational Fluid Dynamics, Phase Contrast Magnetic Resonance Imaging, Vorticity, Cardiac Flow Analysis

\section{Kelvin K L WONG*}

School of Aerospace, Mechanical \& Manufacturing Engineering, and Health Innovations Research Institute (HIRi), RMIT University

\section{Kiao INTHAVONG}

School of Aerospace, Mechanical g Manufacturing Engineering, and Health Innovations Research Institute (HIRi), RMIT University

\section{SUN Zhonghua}

Discipline of Medical Imaging, Department of Imaging and Applied Physics, Curtin University of Technology

\section{Keith LIOW}

School of Aerospace, Mechanical \& Manufacturing Engineering, and Health Innovations Research Institute (HIRi), RMIT University

\section{TU Jiyuan}

School of Aerospace, Mechanical \& Manufacturing Engineering, and Health Innovations Research Institute (HIRi), RMIT University

* The first author who is at the age of 35 or below on the closing date of submission

\section{Introduction}

In this study, we use the right atrium of the heart as an illustration of the flow quantification and visualisation techniques. Specifically, we aim to study the vortices that are generated within the heart chamber. Cardiac flow analysis based on vorticity mapping can define the flow behaviour within the heart chamber $[13,16]$. The first stage of the flow analysis is to be able to generate the flow fields of blood in the cardiac structure based on phase contrast magnetic resonance imaging and then by numerical simulation. The second stage will be to utilise the flow field for vorticity measurement and visualisation. Then, the final stage is to quantify the vorticity field statistically and examine the changes in vorticity levels over time.

Phase contrast magnetic resonance imaging (PC-MRI) belongs to a class of experimental flow imaging by means of quantifying the phase shifts in proton spins of the imaged fluid, and has been widely used to measure flow fields of blood in the cardiac structures in-vivo [5]. Another approach to generate the hemodynamic flow field relies on medical imaging, anatomical reconstruction, and numerical simulation by computational fluid dynamics (CFD). The initial process of this computational framework is based on utilising magnetic resonance or computed tomographic scan images to construct the geometry of the cardiac vessel whereby blood is transported. The next step is to mesh the flow volume and establish the boundary flow conditions, which can be determined by medical imaging measurement, such as Doppler ultrasound or phase contrast magnetic resonance image data [6]. Finally, these conditions can be coupled with a well constructed mesh and a well established computational simulation to generate the flow within the computerised surface reconstruction of the cardiac structure with good accuracy [12].

The flow generation by experimental flow imaging such as PC-MRI and by numerical simulation using CFD have been used in comparative studies for investigation of blood flow in carotid bifurcation [4,6,8,17]. Validation of the CFD simulation in human proximal airways with hyperpolarised 3 He magnetic resonance phase-contrast velocimetry has been performed in vitro to show good agreement between the two techniques [1]. The flow imaging results of the cardiovascular structure in-vivo is often taken as the ground truth data since blood velocities are intrinsically measured with good accuracy. The numerical simulation model is typically used as a predictive tool for generating flow conditions that are difficult to measure. Velocity measurement and mapping systems by PC-MRI is often used to validate computational and predictive frameworks with good reliability. To the best of our knowledge, there is no previous study on the use of PC-MRI and CFD on the right atrial flow and therefore, our work to quantify the vortical flow for this cardiac chamber may open up new insights into the flow behaviour within this anatomy.

In this paper, we aim to present the hemodynamic of blood within a right atrium in order to demonstrate our technique of flow generation, visualisation and comparison. We have detailed the principles of flow measurements based on experimental and computational techniques in Methods. The experimental setup and configuration of the computational platform are outlined in Experiment. Then, we present the results of right atrial flow based on PC-MRI and CFD simulation. Finally, the technical limitations based on the imaging technique and simulation setup is discussed. Future work and contribution of the paper in terms of cardiac flow measurement is presented.

\section{Methods}

The following subsections provide a preliminary background on the techniques of flow field generation by functional medical imaging and 
computational fluid dynamics. This can enhance our technical knowledge of the instruments used in this study.

\section{Phase Contrast Magnetic Resonance Imaging}

Phase contrast magnetic resonance imaging (PC-MRI) is widely used to measure temporal flow fields of up to three spatial dimensions for the purpose of studying flow effects in the heart [3]. It has been able to present flow information pertaining to the cardiovascular system in-vivo. The velocity-encoded phase contrast MRI approach is able to achieve flow measurements of up to three dimensions. This velocity mapping technique is based on the intrinsic sensitivity of proton spins phase in the flow, and enables the acquisition of spatial registered functional information simultaneously with morphological information [5].

In general, 3-dimensional PC-MRI works by registering three separate flow-sensitive volumes in the $x^{-}, y^{-}$and $z$-orientations of the scan. In the quantum mechanical sense, based on the output scan image of an orientation, the flow velocities may be determined by shift of phase at every discrete pixel on which spin phases that pertain to the agglomeration of blood protons are collectively registered. Reconstructing flow vectors using these sets of phase contrast images can be achieved by an appropriate visualisation framework.

\section{Computational Fluid Dynamics}

Numerical models based on CFD is particularly advantageous for situations whereby experimental measurements require further detail, and/or when there is a need to prepare a predictive platform. Clearly, we have shown that cardiac flow analysis is one such case. Yet another potential scenario is the simulation of mechanical structures that are implanted in the human heart. It is worth mentioning that the use of CFD in biomedical engineering has increased markedly as computing technology advances.

In a numerical simulation platform, the governing equations for the viscous, incompressible fluid flow can be written as:

$\frac{\partial \rho}{\partial \mathrm{t}}+(\nabla \cdot V) \rho=0$

$\rho \frac{\mathrm{dV}}{\mathrm{dt}}=\rho g+\mu \nabla^{2} \mathrm{~V}-\nabla \mathrm{P}$

Where $V$ is the fluid velocity vector. $\mu$ is the viscosity, $g$ is the gravitational acceleration, $\rho$ is the density, and $P$ is the dynamic pressure of the fluid.

In order to have a realistic right atrium (RA) model so that the predicted flow would be physiologically correct, the technique of anatomical reconstruction based on medical imaging and processing $[7,9]$ is used. For imaging of the RA, segmentation of the myocardium was performed through a series of time-resolved image slices. The typical imaging instruments for assessment of cardiac chambers or myocardial function include computed tomography and MRI, but MRI is superior to computed tomography since the tissue and blood regions are often well-distinguished in terms of image intensity for boundary definition with MRI. Once the chamber geometry has been created, an appropriate mesh is generated, which effectively discretises the fluid volume in the chamber into a set of discrete cells. The governing equations for the fluid flow (Navier-Stokes equations) are discretised, and the field variables are iterated until a user-imposed convergence criteria is achieved. The iterative behaviour of the numerical solution and to a certain extent the accuracy of the numerical simulations, would depend on the correct imposition of the boundary conditions (inflow and outflow) as well as having a reasonable guess of the solution field at the start of the simulation.

\section{Experiment}

This section describes the experimental procedures that are carried out to prepare the imaging and retrieval of flow information. The computational configuration of the numerical simulation is also presented. The objective of our study is to prepare two sets of results based on the experimental and computational generation of flow fields, and to compare them in terms of flow patterns and magnitudes of their vorticities.

\section{Measurement Slice}

Examination is performed at the section of the heart where the atria are positioned. This section is chosen such that the display of optimal crosssectional area of the right atrium is enabled. A chamber size contains more data points to define the features of interest. The slice section is taken at a location shown in Fig 1 whereby the slice is perpendicular to the axis joining the top of the heart to the apex through the septum. It is effective to base the velocity mapping on the right atrium of the heart using the scan sections that cut through the middle portion of atria. Sectioning of the heart, as shown in the diagram, is used for cardiac flow analysis.

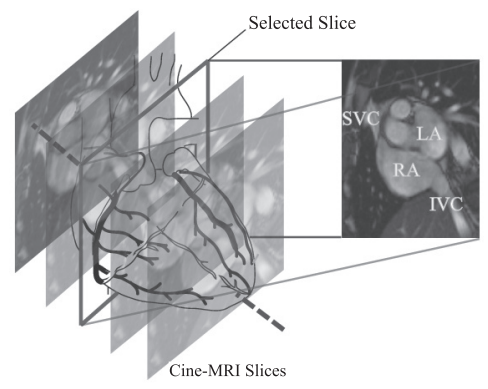

SVC - Superior Vena Cava IVC - Inferior Vena Cava RA - Right Atrium LA - Left Atrium
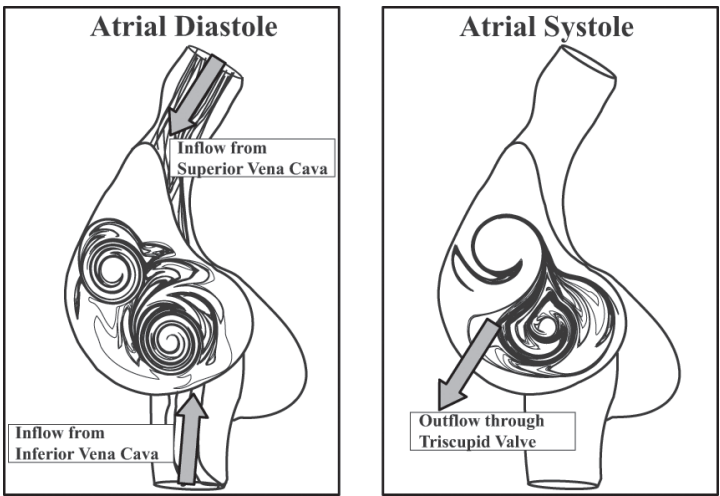

Figure 1 - Sectional View of Blood Flow in the Right Atrium

Inflows from the inferior vena cava (IVC) and superior vena cava (SVC) results in their respective flow diversions that generate two vortices in opposite directions. During atrial diastole, there is maximum suction of blood into the right atrium via the vena cava. The chamber dilates due to the inflow of blood and the closure of the tricuspid valves (TV). We note that the maximum inlet flow at the superior and inferior vena cava is set to be 155 and $128 \mathrm{cms}^{-1}$. At this instance, two strong vortices of opposite rotation are developed. During the systolic phases, the atrium compresses and causes the outflow of blood into the right ventricle which in turn causes the valves to open. We note that one of the vortices becomes significantly weaker than the dominant vortex during their evolution over a cardiac cycle.

\section{Medical Imaging Configuration}

In this study, MRI using the phase contrast imaging protocol was implemented based on the short axis orientation through the atria of the heart. Acquisition of scans pertaining to 25 phases or time frames (based on their indices from $n_{t}=1$ to 25) was completed based on retrospective gating. Our case study subject was a normal male of age 22 years at the time of this experimental scanning. We performed the imaging using the two-chamber view of the right and left atria. The main objective of this imaging is to capture information on the 
large-scale vortices in the right atrium. These vortices can serve as the principle features of the flow structures that we are going to analyse. The acquisition parameters include: echo time $\mathrm{TE}=1.6 \mathrm{~ms}$, repetition time $\mathrm{TR}=47.1 \mathrm{~ms}$, field of view FOV $=(298 \times 340) \mathrm{mm}^{2}$ at a $(134 \times 256)$ pixel matrix, and a velocity encoding (VENC) of $150 \mathrm{cms}^{-1}$. The in-plane resolution of $1.54 \mathrm{~mm} /$ pixel is determined by the pixel spacing and the throughplane resolution that is based on the slice interval is $6 \mathrm{~mm}$.

\section{Numerical Simulation}

The process of creating the CFD mesh of the RA chamber and its related vessels is graphically depicted in Fig 2 . Here, 3D surfaces that were constructed through using the segmented slices of the heart chamber. After processing of the geometry, the final output is a tetrahedral mesh with refinements in the near wall consisting of approximately 800,000 cells. A mesh independence study was carried out to ascertain that the selected domain size is sufficient in resolving the flow gradients. The blood flow through the RA chamber is assumed to be incompressible, homogeneous, laminar and Newtonian. The fluid properties are reported in Table 1. Realising the unsteady nature of the flow field, a transient CFD simulation was performed. The range of Reynolds numbers in the atrial flow throughout one cardiac cycle is dominantly laminar while it may be argued that turbulence can be induced through the curvatures within the geometry. The use of a turbulence model therefore does not necessarily imply greater accuracy in capturing the flow field. Instead the incorrect use of a turbulence model, especially models based on fully developed turbulent flows, will produce an overly diffusive flow because of the additional turbulent viscosity $\mu_{t}$ term. At best, lowReynolds number turbulence models such as the $\mathrm{k}-\epsilon$ and $\mathrm{k}-\omega$ provide improvements over their standard versions. However, in order to fully resolve the any transitional behaviour, the sophisticated LES and Direct Numerical Simulations models need to be used since they are based on the direct Navier-Stokes equations. Therefore in this study, we take a prudent perspective and apply a laminar flow model.

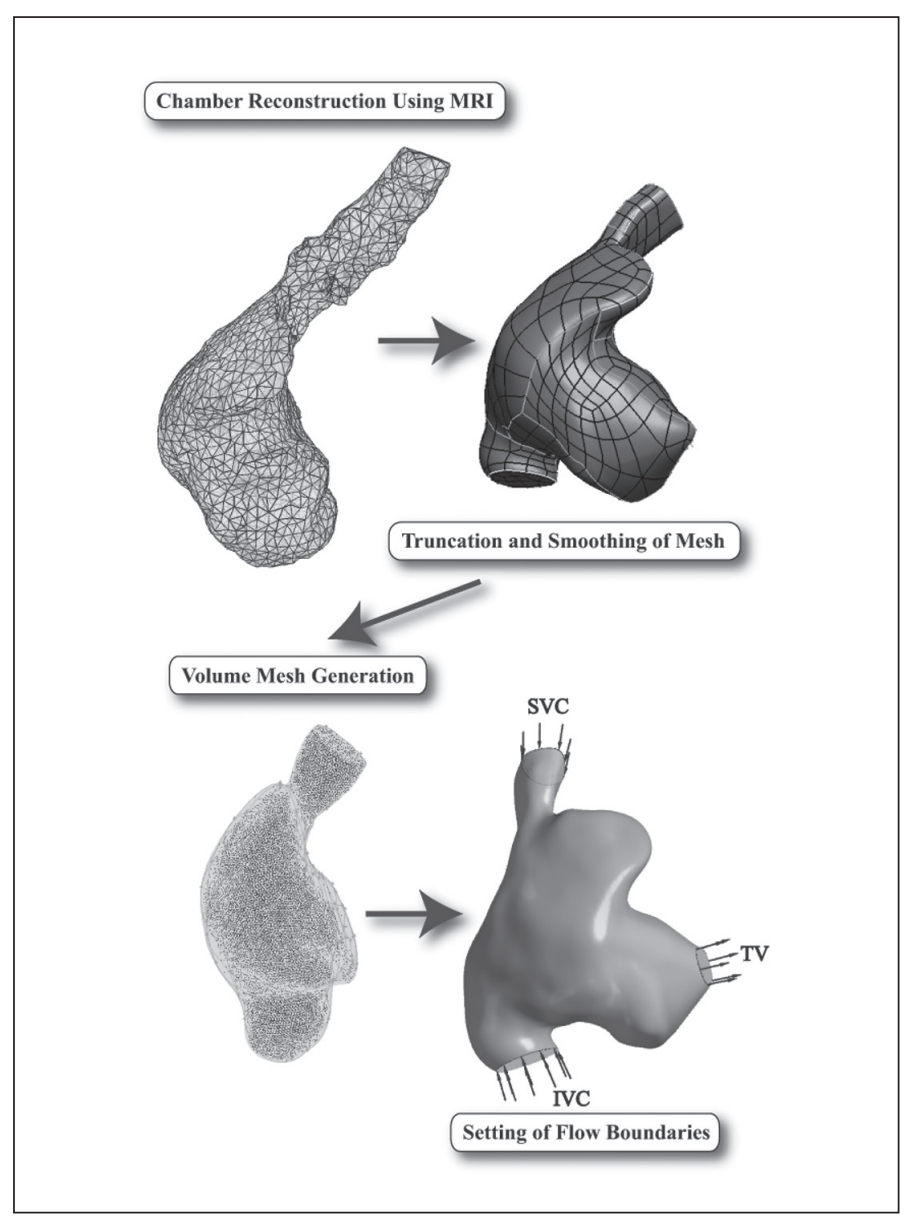

Figure 2 - Stages in Creating a CFD Mesh of the RA Vessel

\begin{tabular}{|c|c|c|}
\hline Parameter & Value & Units \\
\hline Dynamic Viscosity & 0.004 & Pascal \\
\hline Density & 1,176 & $\mathrm{kgm}^{-3}$ \\
\hline Range of IVC (Inflow) Velocities & -1.2200 to 40.1450 & $\mathrm{cms}^{-1}$ \\
\hline Range of SVC (Inflow) Velocities & -0.5120 to 20.3040 & $\mathrm{cms}^{-1}$ \\
\hline Reynolds Number & 518 to 2,738 & \\
\hline
\end{tabular}

Table 1 - Fluid Properties Used in the CFD Simulations

As can be seen in Fig 2, the flow enters the RA chamber through the inferior and superior vena cava (IVC and SVC), and exits through the tricuspid valve (TV). For simplicity, the velocity boundary condition that was applied at the IVC and SVC assumes a uniform spatial profile and has a direction that is normal to the surface. Implementation of a parabolic profile may not be the correct assumption given that the upstream flow may be highly skewed at high velocities. In addition, different degrees of skewness exist for different velocities. Therefore, in order to avoid these complications, a uniformly spaced profile is simply used. For the TV outlet, a transient pressure profile that has zero gradient in the spatial domain is implemented at a virtual outflow environment to ensure that the flow will be constant in direction and velocity at the outlet. Given that the IVC and SVC in-flows are varying throughout the cardiac cycle (Fig 3 and Table 1), therefore defining the inlet boundaries would require a-priori knowledge of the IVC and SVC velocity time histories. Logically, this would be obtained from physical experiments [12-13]. For this study, in-vivo measurements were used to generate the time histories [12].

The flow in the RA chamber is simulated from the end-diastolic state to the early-systole state, ie, corresponding to the normalised time segment of 0.60 to 1.00 in one cardiac cycle (Fig 3). In order to avoid start-up effects from unsteady flows, the flow field was simulated over three periodic cycles where the final flow field predictions were taken at the third cycle. Spatial discretisation of the advection terms in the NavierStokes equations was performed by using a high resolution scheme, while the transient scheme used a second order backward Euler scheme with a time step size of $0.002 \mathrm{~s}$.

While the MRI scans were taken over a complete cardiac cycle of the heart chamber, the scope of the numerical simulations was limited to examining the flow in the RA during the diastole and systole phases. In particular, focusing on particular phases of the cardiac cycle allows the flow analysis to be carried out in the simpler context of an internal flow in a rigid vessel. This is due to the fact that modelling the entire cardiac cycle will require a fluid-structure interaction (FSI) approach and the physical structure of the heart chamber is a dynamic parameter, which makes it much more complicated to simulate the entire cardiac cycle.

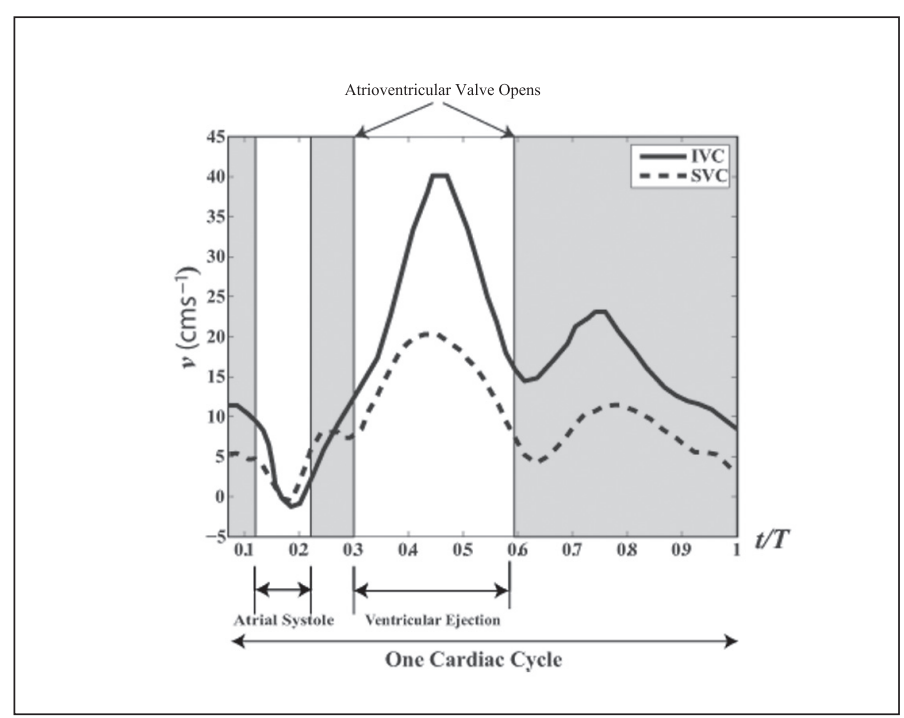

Figure 3 - Plot of Vena Cavae Average Flow Velocities versus Time 
The assumption of rigid boundary walls instead of elastic ones based on the FSI approach allows the simulation to converge and hence produce a set of predicted results. However this comes at the expense of the real physiological cyclic movement of the heart contracting and expanding. In reality, a contraction in the heart will reduce the volume and therefore any cross-sectional area in which the blood flows through. Considering mass conservation that is based on the continuity equation in CFD (Eqn (1)), the contraction is expected to have an effect of intensifying the velocity and vorticity fields. However, if we implement FSI for this simulation, the blood pressure during atrial systole will stretch the vessel elastically and causing the velocity and vorticity fields to attenuate instead.

\section{Results}

The flow results by numerical simulation and phase contrast magnetic resonance imaging are presented in this section. Then, their velocity and vorticity fields are compared in terms of flow pattern developments. Fig 4 shows three snapshots of the CFD flow visualisation in the RA solution domain corresponding to the time instants of $t / T=0.66,0.74$, 0.82 and 0.90 per time cycle of 1.0 . These were selected to match the MRI time frame indices $n_{t}=9,11,13$ and 15 out of a total of 25 frames. Collectively, they form the basis for comparison of the CFD simulations and PC-MRI experiments.

As can be seen, the velocity vectors in the CFD plots are superimposed on the vorticity contours. Here, the velocity vectors are used to depict flow direction on the plane itself, thereby implying a tangential projection. A normalised scale is used for the vector length. For the vorticity contours, the clockwise/anti-clockwise direction of the rotation is provided by using the $y$-axis as a reference axis, while the figure shows the in-plane view of the selected slice. The reader would note that the normal to the slice is almost lying parallel to the y-axis. Finally, it is also worth mentioning that the selection of the plane is chosen as per the MRI plane.

Cardiac flow analysis $[13,14,16]$ that is based on histograms of vorticity field distributions for both modes of flow generation can give a clear overview of their flow differences with some form of quantification.

\section{Discussion}

We note that based on the distribution of CFD and PC-MRI vorticity fields, the difference in their means and standard deviations pertains to an order of zero (Difference in means: $\Delta \omega \leq 2.02 \mathrm{~s}^{-1}$, and difference in standard deviations: $\Delta \sigma \leq 4.07 \mathrm{~s}^{-1}$ for a range of vorticity values at approximately -25 to $+25 \mathrm{~s}^{-1}$ ). The agreement in the CFD simulations and MRI experiments reinforces the evidence of swirling flow in the RA. Although we have further verified PC-MRI flow mapping accuracy using CFD, further testing and comparison of the two modes of flow can be performed based on variation of vorticity interrogation size and resolution of the flow fields in a sensitivity study to understand the effects of flow measurement and visualisation reliability [15]. However, to prevent deviation from the main scope of the paper in terms of flow comparison, such tests may be formulated in the future and further implemented in another specific study.

From the fluid mechanics perspective, blood swirling is caused by the interaction between the two paths of flow from the inlets (IVC and SVC). As can be seen from Fig 1, the generation and suppression of vortex structures in the RA flow is dynamically varying throughout the main cycle. Given that only one dominant vortex has been visualised for the time segment of the cardiac cycle that is presented here, it appears that the presence of the weaker, clockwise vortex can be attributed to the peaking of IVC in-flow during atrial diastole. The potential of using the extensive datasets from the CFD simulations and phase contrast MRI can enable the development of further insight into the vortex structures present in the RA flow. Therefore, applying sophisticated 3D flow visualisation techniques can improve accuracy of comparison for the two techniques.

The Reynolds numbers are typically lower towards end-systole and early diastole due to the slower motion of blood. The blood flow is transitionally turbulent during the onset of systole. The blood inflow velocities at the superior and inferior vena cava tends to increase during the period of systole and causes a collision within the atrium, which generates a turbulent flow that comprise of two large-scale vortices. The range of Reynolds numbers in the atrial flow throughout one cardiac cycle pertains to both laminar and turbulent flows. The simulation results are largely in agreement with the MRI results. In terms of the flow features, the velocity vectors implied that the flow is moving primary in the anticlockwise direction. Partly as a consequence, there is a dominant vortex core-like structure that had remained relatively robust throughout the cardiac cycle. It appears that this dominant vortex has shown a positional dependence on the TV outlet.

In terms of clinical applications, medical imaging modalities such as multislice computed tomography and MRI have been increasingly used to diagnose cardiovascular disease owing to rapid technical developments [10-11]. Despite the improvements in computed tomography, its application in cardiovascular disease is limited to evaluation of the morphological changes of cardiac chambers. On the contrary, functional MR imaging, especially phase-contrast MRI allows for both anatomic and functional assessment of cardiovascular disease [14], and it demonstrates huge potential in the clinical practice. Analysis of hemodynamic flow through the heart will assist clinicians to better understand the formation of thrombosis, and the effect of flow on arterial branches arising from the heart, which will help to achieve the aim of avoiding atherogenicity [2]. Therefore, future studies involving CFD analysis of bilateral heart chambers that include both venous inflow and arterial outflow will be clinically meaningful.

\section{Conclusion}

In this study, we compared the flow pattern developments in a chamber of the heart based on phase contrast magnetic resonance imaging and numerical simulation. For our computational study, we assume the utilisation of static geometry and dynamic flow boundary conditions in a series of surface geometries pertaining to different times of a cardiac cycle. Although the fluid structure interaction of the blood-wall region is neglected, the generated flow fields will not be significantly deviated from the true physiological flow. Experimental study is based on in-vivo magnetic resonance-based flow imaging.

The flow visualisation tools using streamline tracing and vorticity field generation enables the presentation of our results effectively. Vortices exist in three spatial dimensions, but we have simplified our analysis to present two dimensional results for clarity in flow evaluation. Nevertheless, the visualisation of flow patterns remains concise for the two modes of flow field generation.

The comparison of flow results by experimental (PC-MRI) and computational (CFD) approaches shows good qualitative and quantitative agreements. We have demonstrated that the simulation results can be validated using the measured flow from velocity-encoded medical imaging. Our proposed functional analysis using combined phase contrast MRI and CFD is of interest to research into the pathologies or interventions which involve altered relations between form, flow, mobility and timing of the heart.

\section{Acknowledgements}

The financial support provided by the Australian Research Council (ARC project ID DP0986183) is gratefully acknowledged.

\section{Notes}

The first author developed and use a medical image processing software named Medflovan to produce the results displayed in this paper. This is a system entirely created using the $\mathrm{C}++$ object-oriented programming platform to provide cardiac flow visualisation and analysis. Cardiac flow computation and visualisation is performed on a Pentium 4-class processor on a dedicated graphics card with 512 MB of memory. 


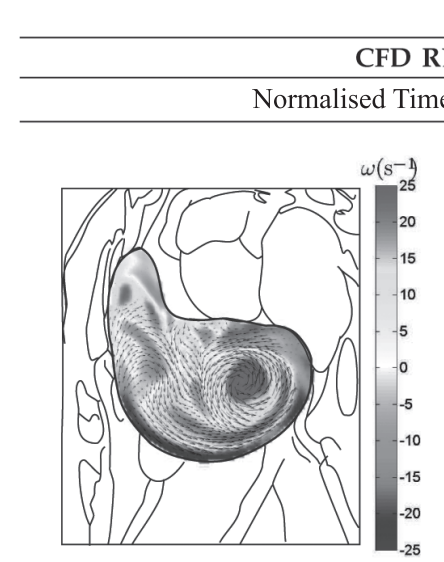

(i) CFD: $t / T=0.66,\left[\begin{array}{l}\bar{\omega} \\ \sigma\end{array}\right]_{\mu}=\left[\begin{array}{c}1.59 \\ 11.15\end{array}\right] \mathrm{s}^{-1}$

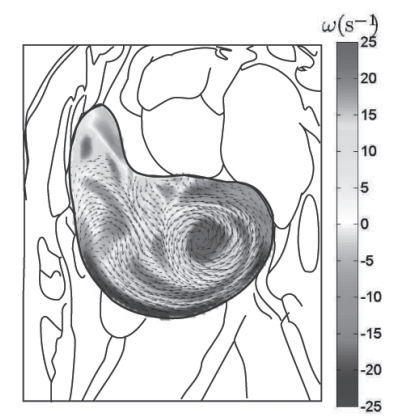

(ii) CFD: $t / T=0.74,\left[\begin{array}{l}\bar{\omega} \\ \sigma\end{array}\right]_{\mu}=\left[\begin{array}{c}1.34 \\ 10.94\end{array}\right] \mathrm{s}^{-1}$

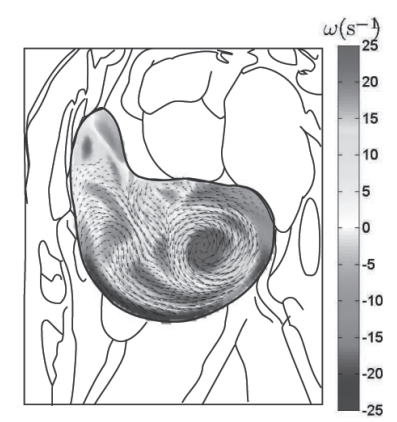

(iii) CFD: $t / T=0.82,\left[\begin{array}{l}\bar{\omega} \\ \sigma\end{array}\right]_{\mu}=\left[\begin{array}{c}1.36 \\ 11.20\end{array}\right] \mathrm{s}^{-1}$

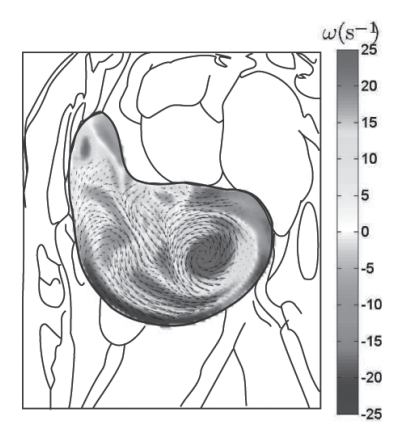

(iv) CFD: $t / T=0.90,\left[\begin{array}{l}\bar{\omega} \\ \sigma\end{array}\right]_{\mu}=\left[\begin{array}{c}1.48 \\ 10.96\end{array}\right] \mathrm{s}^{-1}$
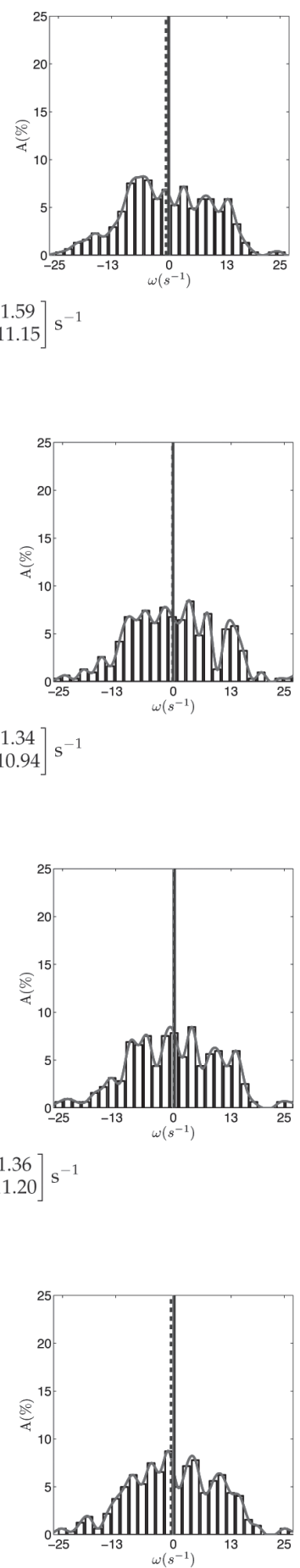
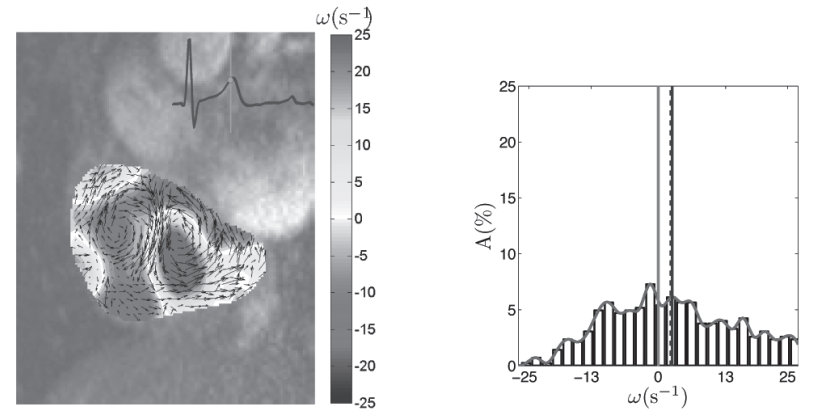

(v) PC-MRI: $n_{t}=9,\left[\begin{array}{l}\bar{\omega} \\ \sigma\end{array}\right]_{\mu}=\left[\begin{array}{c}2.13 \\ 12.64\end{array}\right] \mathrm{s}^{-1}$
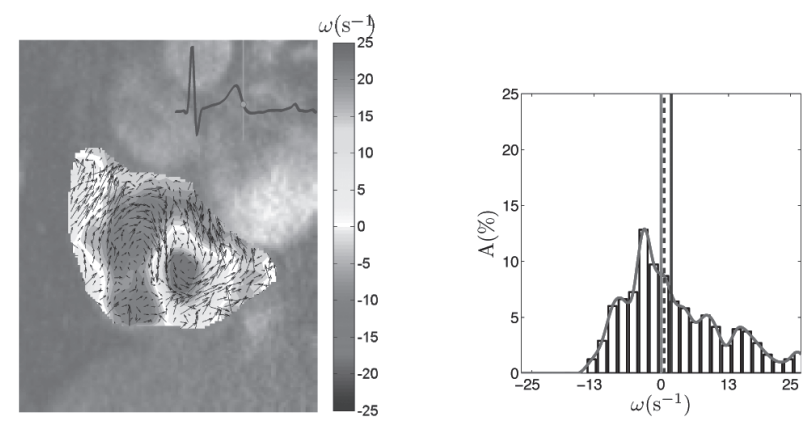

(vi) PC-MRI: $n_{\ddagger}=11,\left[\begin{array}{l}\bar{\omega} \\ \sigma\end{array}\right]_{\mu}=\left[\begin{array}{l}0.02 \\ 9.10\end{array}\right] \mathrm{s}^{-1}$
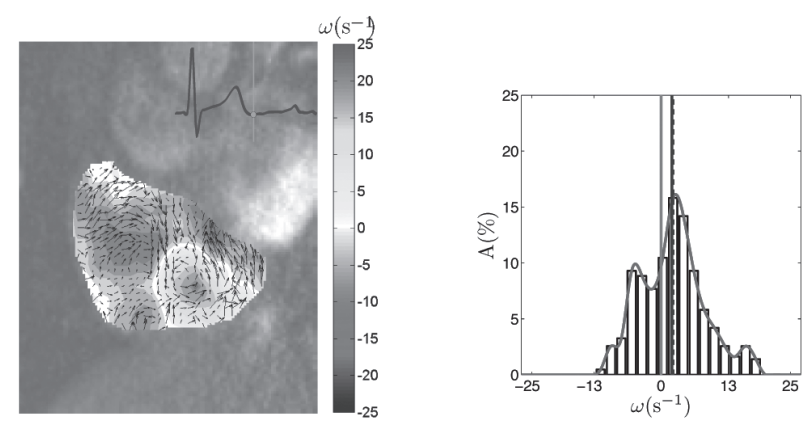

(vii) PC-MRI: $n_{t}=13,\left[\begin{array}{l}\bar{\omega} \\ \sigma\end{array}\right]_{\mu}=\left[\begin{array}{c}-0.6 \\ 7.13\end{array}\right] \mathrm{s}^{-1}$
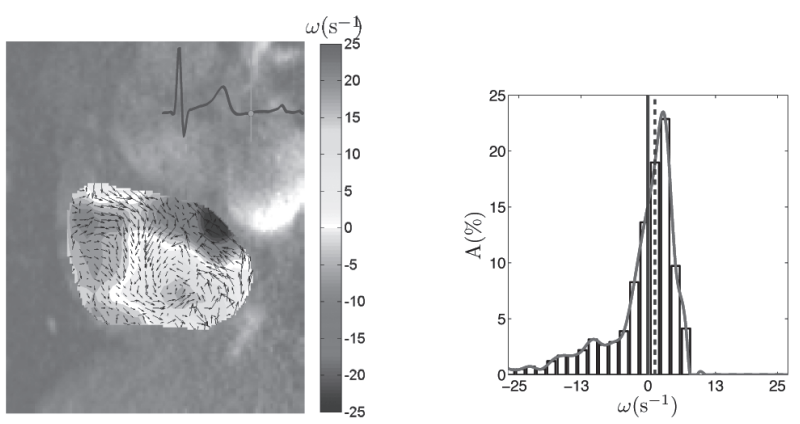

(viii) PC-MRI: $n_{t}=15,\left[\begin{array}{c}\bar{\omega} \\ \sigma\end{array}\right]_{u}=\left[\begin{array}{c}-0.54 \\ 9.40\end{array}\right] \mathrm{s}^{-1}$ 


\section{References}

1. L. de Rochefort, L. Vial, R. Fodil, X. Maitre, B. Louis, D. Isabey, G. Caillibotte, M. Thiriet, J. Bittoun, E. Durand, G. Sbirlea-Apiou, In vitro validation of computational fluid dynamic simulation in human proximal airways with hyperpolarised $3 \mathrm{He}$ magnetic resonance phase-contrast velocimetry. Journal of Applied Physiology. Volume 102, No 5, pp2012-2023. (2007).

2. M. H. Friedman, A. M. Brinkman, J. J. Qin, W. A. Seed., Relation between coronary artery geometry and the distribution of early sudanophilic lesions. Atherosclerosis. Volume 98, pp193-199. (1993).

3. P. J. Kilner, G-Z. Yang, A. J. Wilkes, R. H. Mohiaddin, D. N. Firmin, M. H. Yacoub, Asymmetric redirection of flow through the heart. Nature Medicine. Volume 404, pp759-761. (2000).

4. Q. Long, X. Y. Xu, U. Kohler, M. B. Robertson, I. Marshall, P. Hoskins, Quantitative comparison of CFD predicted velocity and MRI measured velocity fields in a carotid bifurcation phantom. Biorheology. Volume 39, No 3-4, pp467-474. (2002)

5. M. Markl, A. Harloff, T. A. Bley, M. Zaitsev, B. Jung, E. Weigang, M. Langer, J. Hennig, A. Frydrychowicz, Time-resolved 3D MR velocity mapping at 3T: Improved navigator-gated assessment of vascular anatomy and blood flow. Journal of Magnetic Resonance Imaging. Volume 25, pp824-831. (2007).

6. I. Marshall, S. Zhao, P. Papathanasopoulou, P. Hoskins, X. Y. Xu, MRI and CFD studies of pulsatile flow in healthy and stenosed carotid bifurcation models. Journal of Biomechanics. Volume 37, No 5, pp679-687. (2004).

7. R. Merrifield, Q. Long, X. Y. Xu, P. J. Kilner, D. N. Firmin, G-Z. Yang, Combined CFD/MRI analysis of left ventricular flow. Proceedings of the Medical Image and Augmented Reality 2004. pp229-236. (2004).

8. P. Papathanasopoulou, S. Zhao, U. Kohler, M. B. Robertson, Q. Long, P. Hoskins, X. Y. Xu, I. Marshall. MRI measurement of time-resolved wall shear stress vectors in a carotid bifurcation model, and comparison with CFD predictions. Journal of Magnetic Resonance Imaging. Volume 17, No 2, pp153-162. (2003).

9. N. R. Saber, A. D. Gosman, N. B. Wood, P. J. Kilner, C. L. Charrier, D. N. Firmin Computational flow mdelling of the left ventricle based on in-vivo MRI data. Annals of Biomedical Engineering. Volume 29, pp275-283. (2001).

10. T. Sommer, M. Hackenbroch, U. Hofer, A. Schmiedel, W. A. Willinek, S. Flacke, J. Gieseke, F. Traber, R. Fimmers, H. Litt, H. Schild, Coronary MR angiography at $3.0 \mathrm{~T}$ versus that at $1.5 \mathrm{~T}$ : initial results in patients suspected of having coronary artery disease. Radiology. Volume 234, pp718-725. (2005).

11. Z. Sun, K. H. Ng, Multislice CT angiography in cardiac imaging-part II: Clinical applications in coronary artery disease. Singapore Medical Journal. Volume 51, pp282-289. (2010)

12. L. Wexler, D. H. Bergel, I. T. Gabe, G. S. Makin, C. J. Mills, Velocity of blood flow in normal human venae cavae. Circulation Research. Volume 23, pp349359. (1968).

13. K. K. L. Wong, R. M. Kelso, S. G. Worthley, P. Sanders, J. Mazumdar, D. Abbott, Cardiac flow analysis applied to phase contrast magnetic resonance imaging of the heart. Annals of Biomedical Engineering. Volume 37, No 8, pp1495-1515. (2009)

14. K. K. L. Wong, R. M. Kelso, S. G. Worthley, P. Sanders, J. Mazumdar, D. Abbott, Noninvasive cardiac flow assessment using high speed magnetic resonance fluid motion tracking. PLOS ONE. Volume 4, No 5, p5688. (2009).

15. K. K. L. Wong, J. Y. Tu, R. M. Kelso, Vortical flow analysis. Journal of Mechanics in Medicine and Biology. Volume 10, No 2, pp1-23. (2010).

16. K. K. L. Wong, J. Y. Tu, R. M. Kelso, S. G. Worthley, P. Sanders, J. Mazumdar, D. Abbott, Cardiac flow component analysis. Medical Engineering \& Physics. Volume 32, No 2, pp174-188. (2010).

17. S. Z. Zhao, P. Papathanasopoulou, Q. Long, I. Marshall, X. Y. Xu, Comparative study of magnetic resonance imaging and image-based computational fluid dynamics for quantification of pulsatile flow in a carotid bifurcation phantom. Annals of Biomedical Engineering. Volume 31, No 8, pp962-971. (2003).

\section{About the Authors}

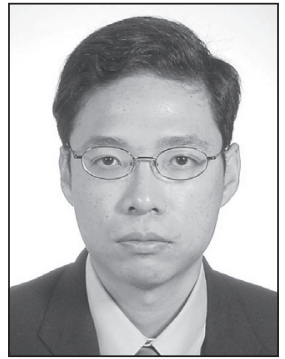

Kelvin K L WONG PhD Mappl IT BEng (Hons) Email: k.wong@rmit.edu.au

Dr Wong received his BEng degree from Nanyang Technological University, MAppIIT from the University of Sydney, and PhD degree from the University of Adelaide. He is currently a Research Fellow of biomedical engineering at RMIT University performing research and development of new medical image processing techniques and medical image velocimetry systems for the study of cardiovascular flow. Dr Wong's research interests are biofluid mechanics, medical imaging, image processing and flow visualisation.

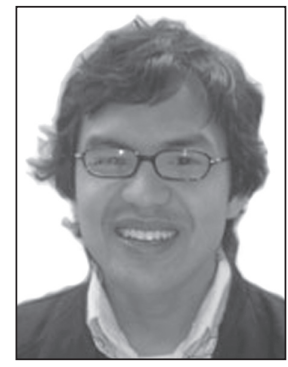

Kiao INTHAVONG BEng PhD

Email: inthavong@rmit.edu.au

Dr Inthavong received his bachelor's degree in Mechanical Engineering from Swinburne University, Australia in 2003. He is a Research Fellow at RMIT studying biomedical flows, gas-particle flows in the respiratory airways, HVAC systems in buildings and particle dispersion for indoor air. His research interests include heat transfer, computational fluid dynamics (CFD), numerical heat transfer, multiphase flows and biomedical applications.

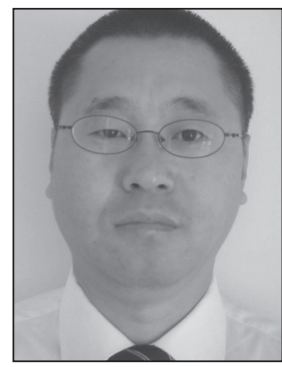

\section{SUN Zhonghua MB PhD}

Email: z.sun@curtin.edu.au

Dr Sun obtained his MB and PhD degrees in clinical medicine and medical imaging from Harbin Medical University in China and University of Ulster, the UK respectively. He worked as a radiologist in the department of radiology at Peking Union Medical College Hospital for nine years prior to taking up oneyear medical fellowship during 1998-1999 in Japan. He is currently a Senior Lecturer in medical imaging at Curtin University. His research interests include 3D medical image visualisation and processing, virtual reality and simulation in medicine and hemodynamic analysis of cardiovascular diseases.

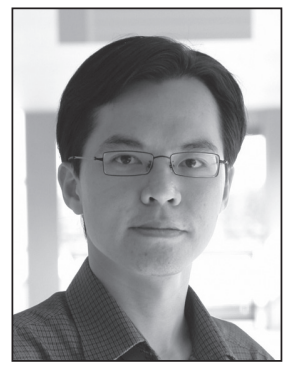

\section{Keith LIOW BEng PhD}

Email: keith.ys.liow@gmail.com

Dr Liow is a CFD Specialist with VPAC, and works as a RGD Consulting Engineer in industry. He received his BEng and PhD degrees in Mechanical Engineering at Monash University. Prior to his current position, he was a lecturer in Mechanical Engineering for five years at Monash University (Malaysian campus). His current research interests include aerodynamics, HVAC and bio-fluid mechanics.

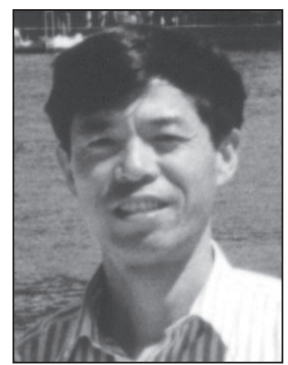

TU Jiyuan PhD MS BEng (Hons)

Email: Jiyuan.Tu@rmit.edu.au

Prof Tu obtained his BEng and MEng degrees from Northeast University, China in 1982 and 1984, and his PhD degree from the Royal Institute of Technology, Stockholm, Sweden in 1992 respectively. He is currently a Professor and Deputy Head (Research and Innovation) of the School of Aerospace, Mechanical and Manufacturing Engineering at the RMIT University, Melbourne, Australia. His research interests focus on computational fluid dynamics (CFD) and its applications in heat transfer, industry multiphase flows, and biomedical engineering. Prof Tu is a recipient of many prestigious fellowship awards including an Australian-American Fulbrigh Senior Scholar award, Japan Society for the Promotion of Science Fellowship and Korea Science and Engineering Foundation Fellowship. He is one of the recipients for the prestigious Brennan Medal for the best book in 2009 awarded by the Institution of Chemical Engineers (IChemE). He has over 200 international refereed publications in areas of his expertise and published a textbook for undergraduate teaching in CFD by Elsevier. He is serving as one of the Editors for Journal of Computational Multiphase Flows published by Multi-Science (the UK). 